\title{
Non-alcoholic fatty liver disease: a new epidemic in children
}

\author{
Mirta Ciocca, M.D., ${ }^{a}$ Margarita Ramonet, M.D., ${ }^{b}$ and Fernando Álvarez, M.D. ${ }^{c}$
}

\begin{abstract}
Non-alcoholic fatty liver disease is considered one of the most common causes of liver disease in adults and children, consistent with the increased prevalence of obesity in both populations worldwide. It is a multifactorial condition involving a broad spectrum of liver diseases than range from simple steatosis to steatohepatitis, and characterized by histological findings of inflammation and fibrosis. Its pathogenesis and progression are not fully understood yet, and a more complete understanding of liver disease may aid in developing new therapies and noninvasive diagnostic tools. Liver biopsy remains the gold standard for disease staging. Although lifestyle and diet modifications are the keys in non-alcoholic fatty liver disease treatment, the development of new drugs may be promising for patients failing first-line therapy.

Key words: non-alcoholic fatty liver disease, childhood obesity, hepatic steatosis.
\end{abstract}

a. Division of Hepatology and Pediatric Liver Transplant Hospital Alemán. Buenos Aires.

b. Member of the National Committee of Hepatology, Argentine Society of Pediatrics (Sociedad Argentina de Pediatría, SAP).

Member of the Standing Committee of the International Pediatric Association (IPA).

c. Department of Pediatrics. CHU Sainte-Justine. University of Montreal. Canada.

E-mail address:

Mirta Ciocca, M.D., mciocca@intramed.net

Funding:

None.

Conflict of interest: None.

Received: 3-18-2016 Accepted: 5-04-2016 http:/ /dx.doi.org/10.5546/aap.2016.eng.563

\section{INTRODUCTION}

Non-alcoholic fatty liver disease (NAFLD) is defined by hepatic fat infiltration of more than $5 \%$ of hepatocytes, in the absence of alcohol intake, or viral, autoimmune, metabolic, drug-induced or other type of liver disease; whereas non-alcoholic steatohepatitis (NASH) is also characterized by an histopathological pattern of inflammation or fibrosis. ${ }^{1-3}$

\section{Epidemiology}

Over the past three decades, NAFLD has been considered one of the most common causes of liver disease in adults and children, consistent with the increased prevalence of obesity in both populations. ${ }^{4}$

According to the World Health Organization (WHO), obesity is a multifactorial and complex chronic disease occurring when calories consumed exceed energy expenditure over a long enough period. It usually develops during childhood or adolescence, and is characterized by an increase in body fat, whose size and distribution have an impact on the subject's health.

Obesity is the most common nutritional disorder in developed countries. Its overall prevalence is 16\% among children aged between 6 and 12 years old. Over the past decades, it has increased two- to fourfold.

Preschool obese children are five times more likely to be overweight during adolescence. Forty percent of 7-year-old children and $70 \%$ of adolescents with obesity become obese adults. ${ }^{5}$

The prevalence of NAFLD is affected by the interaction of several genetic and environmental factors. It is strongly related to environmental determining factors, especially in the family environment, which is where factors affecting energy balance in young children originate. Parents play a very important role in obesity etiology: socioeconomic level, education level, parental employment status, overweight, intrafamilial relationships, intra-familial communication, food choice, and physical activity.

Overweight and obesity have become a generalized problem worldwide, especially in Latin America, and above all, in North America and the Caribbean, where $23 \%$ of adults and $7 \%$ of preschool children are overweight. ${ }^{6}$

In that region, Caribbean countries have the highest prevalence among adults. The countries with the highest obese population are Saint Kitts and Nevis (40.9\%), followed by The Bahamas (35\%), and Barbados (33.4\%). In Latin America, Mexico is the country with the highest obesity 
rate, affecting $32.8 \%$ of adults; followed by Venezuela with $30.8 \%$, Argentina with $29.4 \%$, and Chile with 29.1\%. In 2013, more than 42 million children younger than 5 years of age worldwide were overweight; of them, more than $80 \%$ lived in developing countries. ${ }^{7}$

What is the cause of the increasing rates of childhood overweight and obesity over the past years?

The worldwide increase in childhood obesity is the result of multiple factors, especially the following:

- There is an increased consumption of low-cost, industrial food rich in fat, sugar and salt, but poor in fiber and other nutrients.

- Fruit, fresh vegetables and other highlynutritious food are costly.

- Corporations implement strategies to increase access to and availability of processed food and marketing actions aimed at promoting unhealthy food and sugar-sweetened beverages consumption during childhood.

- The level of physical activity has decreased due to a more sedentary lifestyle and urban life characteristics. ${ }^{7}$

Due to the increase in obesity, between $20 \%$ and $30 \%$ of adults and between $3 \%$ and $10 \%$ of children, at a 2:1 male:female ratio (Occidental countries), may develop NAFLD. They account for approximately $70-80 \%$ of the obese child population. ${ }^{8}$

In a study conducted in San Diego (USA), out of 742 autopsies done between 1993 and 2003 in the 2- to 19 -year-old population, $9.6 \%$ had NAFLD, which increased to $38 \%$ among obese children. In relation to ethnicity, the following percentages were observed: Hispanic, $12 \%$; Asian, 10.2\%; Anglo-Saxon, 8.6\%; and African American, $1.5 \%{ }^{9}$

NAFLD prevalence increases with age; it is more common among children aged 11 to 13 years old, in association with hormonal changes and the development of peripheral insulin resistance. ${ }^{10}$

Feldstein et al., in a study on the natural history of NAFLD conducted over a 20-year period in a population of 66 children, whose average age was $13.9 \pm 3.9$ years old, found that four children having NAFLD progressed to cirrhosis and end-stage liver disease and required a liver transplant. ${ }^{11}$

\section{Pathogenesis}

NAFLD pathogenesis and progression are not fully understood yet. It has been typically considered a two-stage disease. During the first stage, fat accumulates in the liver (steatosis), resulting in hyperinsulinemia and insulin resistance, influenced by genetic susceptibility, epigenetic mechanisms, a sedentary lifestyle, and a high-calorie diet. Steatosis is the consequence of an increased release by adipose tissue, an increased lipogenesis, and an altered fatty acid metabolism. The accumulation of triglycerides in the liver mostly results from adipose tissue lipolysis (60\%) and hepatic de novo lipogenesis $(26 \%)$, and their dietary contribution in terms of chylomicrons is minimal (14\%).

Lipid accumulation in the liver exacerbates insulin resistance by interfering with the phosphorylation of insulin receptors. This leads to the second stage, which is characterized by the production of oxidative stress, mitochondrial dysfunction, altered proinflammatory cytokine levels, and stellate cell activation. These processes lead to necrosis, inflammation, and intrahepatic fibrosis.

Recently, it has been proposed that an affected microbiota and increased intestinal patency deepen liver exposure to liver-derived bacterial products, such as lipopolysaccharides. These products stimulate innate immune system receptors, leading to the activation of the inflammatory process and hepatic fibrogenesis.

Hepatic stellate cells are considered the main source of the extracellular matrix during NASH development. In addition, the hepatic progenitor cell compartment is expanded in children having NAFLD, and its activation is involved in liver response to oxidative stress, disease progression, and fibrosis development.

Adipocytokines are involved in simple steatosis progression to NASH. Adipocytes or inflammatory cells (mainly macrophages), which infiltrate adipose tissue during insulin resistance, are responsible for adipocytokine secretion. Adipose tissue expansion, particularly visceral fat, is associated with a reduced release of antiinflammatory cytokines and an increased level of proinflammatory molecules.

Over the past years, the pathophysiological theories of NAFLD have been supplemented with the discovery of new genetic polymorphisms, which may promote the development of NAFLD and its progression to NASH. Each polymorphism is involved in a different stage and, in the future, this disease pathophysiology may be considered a "multiple stage hypothesis". 1,8,12-14 


\section{Clinical presentation}

Most children with NAFLD have no symptoms. Some have non-specific symptoms such as abdominal pain and fatigue. The physical exam shows an increased waist circumference, stretch marks, and acanthosis nigricans on the neck, armpit and in skin folds, a typical sign of insulin resistance. Liver enlargement may be detected in $50 \%$ of cases, whereas spleen enlargement is less common and associated with advanced liver disease.

Alanine transaminase (ALT), alkaline phosphatase, and gamma-glutamyl transferase levels may be high. Insulin resistance, dyslipemia, and hyperuricemia may serve as guiding parameters for metabolic syndrome. . $2,15,16^{2}$

Waist circumference measurement (measured at the edge of the iliac crests) is an important anthropometric parameter to identify central fat accumulation and, if increased, it may predict the risk of insulin resistance and metabolic syndrome.

Body mass index (BMI) is a simple, lowcost, easily accessible tool to determine the weight to height ratio commonly used to identify overweight and obesity. ${ }^{8}$

The sophistication of this multifactorial process and the variety in NAFLD histopathological findings hinder efforts to design an adequate therapeutic intervention.

Using a pediatric algorithm may help to standardize diagnostic criteria and a timely referral of these patients ${ }^{4}$ (Figure 1 ).

\section{Diagnosis}

NAFLD should be suspected in overweight or obese patients with compatible ultrasound findings or a high ALT level (twice or thrice the upper limit of normal). However, an ALT finding is not sensitive or specific to NAFLD, given that its value may fluctuate and even be normal in patients with NAFLD, NASH, or cirrhosis. . $^{8,12,17}$

A liver biopsy is the gold standard for NAFLD diagnosis and helps to differentiate simple steatosis from NASH, establish the degree of fibrosis, and make a differential diagnosis. However, it is an invasive, relatively costly procedure associated with an uncommon though significant number of complications. In addition, it is necessary to consider the sampling error because it accounts for a minimal portion of the organ (approximately 1/50 000), and an unevenly distributed disease. . $^{1-3,8}$

Given the high prevalence of NAFLD, and the limitations and difficulties related to liver biopsy, it is necessary to develop non-invasive diagnostic modalities. To this end, numerous biomarkers have been studied, but none has been accurate enough so far. ${ }^{7,8,12,16}$

Liver ultrasound is one of the most commonly diagnostic imaging tests used; it is the initial test done in patients suspected of NAFLD. It is a very attractive study because there is no radiation involved. However, it is not effective to detect mild steatosis (approximately $<20 \%$ of fat infiltration), so a normal result does not rule out NAFLD. In addition, it depends on the operator and interpreter, with a suboptimal intra- and inter-observer agreement. Ultrasound effectiveness is limited in morbidly obese patients. ${ }^{12-16}$

A computed tomography (CT) may help to diagnose hepatic steatosis. A CT is more specific than an ultrasound, but it is not recommended for routine use because of radiation exposure. . $2,15-18^{-18}$

A magnetic resonance imaging (MRI) may provide a quantitative measure of infiltrated fat, but few studies have investigated its use in the pediatric population. An MRI may detect up to $3 \%$ of steatosis and has a lower variability, making it more sensitive than an ultrasound. The limitations of an MRI include its high cost, a prolonged scanning time, and the frequent need for sedation in young children. ${ }^{12,16,18-20}$

The magnetic resonance spectroscopy is the more accurate imaging technique for steatosis detection and quantification. In adults, it has demonstrated to have a $100 \%$ sensitivity and a $97 \%$ specificity. However, this method poses the same limitations as an MRI, and requires specific equipment, which is available only in specialized facilities. ${ }^{10,12,16}$

An elastography is a technique that has been approved for adults by the US Food and Drug Administration (FDA). It consists in measuring the propagation speed of elastic waves through the liver and helps to assess the degree of liver fibrosis in a non-invasive manner. Ongoing research studies will allow to establish their usefulness in pediatric patients. ${ }^{10,12,16}$

\section{Differential diagnosis}

An increased transaminase level in an overweight or obese patient does not account for a NAFLD/NASH diagnosis. It is necessary to rule out other muscle or liver diseases before making such diagnosis in children or adolescents.

In general, NAFLD does not occur in very young children $(<3$ years old), and is rare in 
children younger than 10 years old. To make a differential diagnosis, the clinical presentation should be considered first of all, then humoral findings, and finally, liver biopsy.

The presence of microvesicular steatosis should be considered a warning sign of the following potential diagnoses: mitochondrial diseases, disorders of fatty acid oxidation, urea cycle disorders, lysosomal acid lipase deficiency
(Wolman disease and cholesteryl ester storage disease), and hepatotoxicity caused by sodium valproate. In the case of chronic liver disease with hepatic steatosis that has to be differentiated from NAFLD/NASH, it is necessary to consider Wilson's disease, autoimmune hepatitis, chronic hepatitis C, cystic fibrosis, alpha-1 antitrypsin deficiency, etc. Table 1 shows the most common differential diagnoses in this condition. ${ }^{12,16,18}$

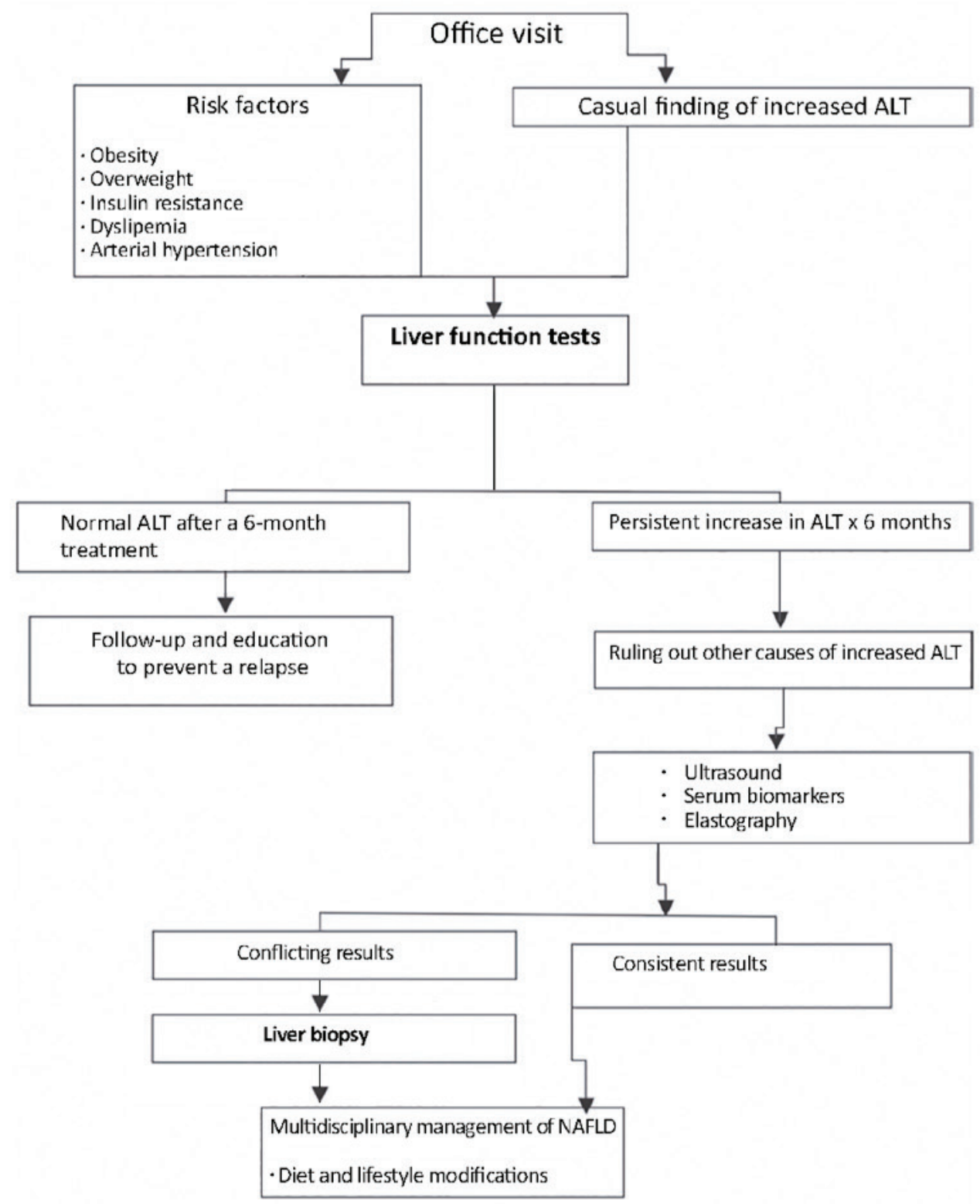

ALT: alanine transaminase; NAFLD: non-alcoholic fatty liver disease. 


\section{Treatment}

Considering that NAFLD may progress to advanced liver disease and cirrhosis, patients should receive early treatment.

\section{- Diet and lifestyle modifications}

The main goal of treatment is implementing lifestyle modifications for a progressive weight loss. A fast weight loss may accelerate the liver inflammatory process. By reducing food intake and increasing physical activity, patients may lose weight gradually. It has been demonstrated that a 5\% BMI reduction is associated with significant changes in waist circumference and decreased cholesterol, triglyceride, and insulin resistance levels in children, and reduced liver enzymes in adults. The impact on insulin resistance is associated with improvements in NAFLD. Also, after 24 months of sustaining lifestyle modifications, histological findings improve significantly.

A pediatric diet should be adequately balanced to promote a harmonious development. Recommendations include reducing the intake of fast-release carbohydrates (fructose), not only to improve insulin resistance and reduce lipogenesis, but also to counteract the fibrogenic and proinflammatory effects of fructose.

Parental involvement is critical for the successful management of childhood obesity. It has been demonstrated that modifying family behavior should be the main goal in the treatment of obese children.

Future studies will probably define the necessary level of weight loss in children for a significant histological improvement and the most adequate diet to achieve such goal. $8,12,14,17,21-23$

\section{- Drug treatment}

Drug treatment for NAFLD has been under investigation for the past years. But none of the studied drugs has proven useful in pediatrics.

Patients having NASH and diabetes are at a higher risk for a more aggressive liver disease. Among the drugs that increase insulin sensitivity in pediatric patients, only metformin has been studied, which has been safe and effective to treat childhood diabetes. Metformin is not recommended in non-diabetic patients, outside the clinical research field.

Given that oxidative stress is considered to play a potential role in NASH development, a series of antioxidants has been studied as possible treatments. A randomized, controlled study conducted in pediatric patients comparing vitamin $C$, vitamin $E$, and placebo treatments demonstrated that treatment for two years in NAFLD patients was not better than diet and exercise in terms of histological improvement.

Considering the potential role of the microbial flora in NAFLD pathogenesis, changes in the gut microbiota may be useful for the prevention and development of steatohepatitis. Preliminary results from studies using prebiotics and probiotics are promising. ${ }^{8,12,14,16,23}$

\section{- Bariatric surgery}

Bariatric surgery may reduce the level of steatosis, liver inflammation, and fibrosis in NAFLD. Roux-en-Y gastric bypass is a safe and effective procedure for extremely obese adolescents. It should be considered for selected patients who have a BMI $>40 \mathrm{~kg} / \mathrm{m}^{2}$ and severe comorbidities, such as NAFLD, or a BMI $>50 \mathrm{~kg} / \mathrm{m}^{2}$ and less severe morbidities. In the pediatric field, its indication should consider the patient's physical and emotional maturity, his/her personal desire to have the procedure, prior weight loss attempts, and his/her ability to comply with the necessary follow-up care. ${ }^{24,25}$

TABLE 1. Causes of hepatic steatosis in children

\begin{tabular}{|c|c|c|c|}
\hline Genetic/metabolic & Nutritional & Systemic & Pharmacological \\
\hline Alpha-1 antitrypsin deficiency & Obesity & Autoimmune hepatitis & Glucocorticoids \\
\hline Lysosomal acid lipase deficiency & Total parenteral nutrition & Celiac disease & Methotrexate \\
\hline Congenital disorder of glycosylation & & Cystic fibrosis & Toxins (alcohol, \\
\hline Disorders of fatty acid oxidation & & Type 1 diabetes & pesticides, etc.) \\
\hline Galactosemia & & Hepatitis C & Valproate \\
\hline Glycogen storage disease & & Inflammatory intestinal disease & \\
\hline Fructosemia & & Reye syndrome & \\
\hline \multicolumn{4}{|l|}{ Type 1 tyrosinemia } \\
\hline Wilson's disease & & & \\
\hline
\end{tabular}




\section{CONCLUSIONS}

- NAFLD is the most common cause of chronic liver disease in children and adolescents.

- Its early detection and management are critical to prevent progression to end-stage liver disease.

- Overweight and obesity have now become an epidemic as a result of a basically sedentary lifestyle, inadequate physical activity, and a high-calorie, imbalanced diet.

- Governments, international associations, scientific societies, the civil society, nongovernmental organizations, and the private sector play a crucial role in the prevention of obesity and, therefore, NAFLD.

- Pediatricians play a key role in the timely recognition of this disease and in the implementation of adequate measures aimed at its prevention.

\section{REFERENCES}

1. Tiniakos DG, Vos MB, Brunt EM. Nonalcoholic fatty liver disease: pathology and pathogenesis. Annu Rev Pathol 2010;5:145-71.

2. Brunt EM. Pathology of nonalcoholic fatty liver disease. Nat Rev Gastroenterol Hepatol 2010;7(4):195-203.

3. Schwimmer JB, Behling C, Newbury R, Deutsch R, et al. Histopathology of pediatric nonalcoholic fatty liver disease. Hepatology 2005;42(3):641-9.

4. Alisi A, Feldstein AE, Vilani A, Raponi M, et al. Pediatric nonalcoholic liver disease: a multidisciplinary approach. Nat Rev Gastroenterol Hepatol 2012;9(3):152-61.

5. Nader PR, O'Brien M, Houts R, Bradley R, et al. Identifying risk for obesity in early childhood. Pediatrics 2006;118(3):e594-601.

6. Organización de las Naciones Unidas para la Alimentación y la Agricultura (FAO). Panorama de la Seguridad Alimentaria y Nutricional en América Latina y el Caribe 2013. [Accessed on: May $5^{\text {th }}, 2016$. Available at: http:// www.fao.org/docrep/019/i3520s/i3520s.pdf.

7. World Health Organization. Obesity and overweight. Geneva:WHO;2015. [Accessed on: May $5^{\text {th }}, 2016$. Available at: http:/ /www.who.int/mediacentre/factsheets/fs311/ en/.

8. Vajro P, Lenta S, Socha P, Dhawan A, et al. Diagnosis of nonalcoholic fatty liver disease in children and adolescents: position paper of the ESPGHAN Hepatology Committee. J Pediatr Gastroenterol Nutr 2012;54(5):700-13.

9. Schwimmer JB, Deutsch R, Kahen T, Lavine JE, et al. Prevalence of fatty liver in children and adolescents.
Pediatrics 2006;118(4):1388-93.

10. Feldstein AE, Charatcharoenwitthaya P, Treeprasertsuk $\mathrm{S}$, Benson JT, et al. The natural history of non-alcoholic fatty liver disease in children: a follow-up study for up to 20 years. Gut 2009;58(11):1538-44.

11. Berardis S, Sokol E. Pediatric non-alcoholic fatty liver disease: an increasing public health issue. Eur J Pediatr 2014;173(2):131-9.

12. Fusillo S, Rudolph B. Nonalcoholic fatty liver disease. Pediatr Rev 2015;36(5):198-205.

13. Marzuillo P, Grandone A, Perrone L, Miraglia del Giudice E. Understanding the pathophysiological mechanisms in the pediatric non-alcoholic fatty liver disease: the role of genetics. World J Hepatol 2015;7(11):1439-43.

14. Roberts EA. Pediatric nonalcoholic fatty liver disease (NAFLD): a"growing" problem?J Hepatol 2007;46(6):1133-42.

15. Shannon A, Alkhouri N, Carter-Kent C, Monti L, et al. Ultrasonographicquantitative estimation of hepatic steatosis in children with NAFLD. J Pediatr Gastroenterol Nutr 2011;53(2):190-5.

16. Feldstein AE, Patton-Ku D, Boutelle KN. Obesity, nutrition and liver disease in children. Clin Liver Dis 2014;18(1):219-31.

17. Mann JP, Goonetilleke R, McKiernan P. Paediatric nonalcoholic fatty liver disease: a practical overview for nonspecialists. Arch Dis Child 2015;100(7):673-7.

18. Marzuillo P, Grandone A, Perrone L, Miraglia del Giudice E. Controversy in the diagnosis of pediatric non-alcoholic fatty liver disease. World J Gastroenterol 2015;21(21):6444-50.

19. Vos MB. Is it time to advance pediatric NAFLD diagnosis to the magnetic resonance imaging era? Hepatology 2015;61(6):1779-80.

20. Schwimmer JB, Middleton MS, Behling C, Newton KP, et al. Magnetic resonance imaging and liver histology as biomarkers of hepatic steatosis in children with nonalcoholic fatty liver disease. Hepatology 2015;61(6):1887-95.

21. KootBG, van derBaan-Slootweg $\mathrm{OH}$,Tamminga-Smeulders $\mathrm{CL}$, Rijcken $\mathrm{TH}$, et al. Lifestyle intervention for nonalcoholic fatty liver disease: prospective cohort study of its efficacy and factors related to improvement. Arch Dis Child 2011;96(7):669-74.

22. De Bruyne RM, FitzpatrickE, Dhawan A. Fatty liver disease in children: eat now pay later. Hepatol Int 2010;4(1):375-85.

23. Gibson PS, Lang S, Gilbert M, Kamat D, et al. Assessment of diet and physical activity in paediatric non-alcoholic fatty liver disease patients: a United Kingdom case control study. Nutrients 2015;7(12):9721-33.

24. Vos MB. NAFLD in the transition from adolescence to young adulthood. Clin Liver Dis 2014;4(4):93-5.

25. Nobili V, Vajro P, Dezsofi A, Fischler B, et al. Indications and limitations of bariatric intervention in severely obese children and adolescents with and without nonalcoholic steatohepatitis:ESPGHANHepatology CommitteePosition Satatement. J Pediatr Gastroenterol Nutr 2015;60(4):550-61. 